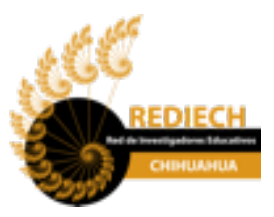

Red de Investigadores Educativos Chihuahua A.C. Chihuahua, México www.rediech.org

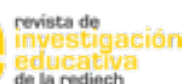

de la rediech

ISSN: 2007-4336

ISSN-e: $2448-8550$

http://www.rediech.org/ojs/2017/index.php/ie rie rediech/index

Antonio Favila Tello

Plinio Hernández Barriga

2019

\title{
LA DESIGUALDAD EDUCATIVA EN MICHOACÁN MEDIDA A TRAVÉS DEL COEFICIENTE DE GINI
}

IE Revista de Investigación Educativa de la REDIECH, 10(19), pp. 139-153.

DOI: http://dx.doi.org/10.33010/ie_rie_rediech.v10i19.646

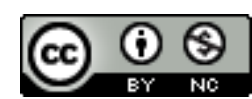

Esta obra está bajo licencia internacional

Creative Commons Reconocimiento-NoComercial 4.0.

CC BY-NC 4.0 


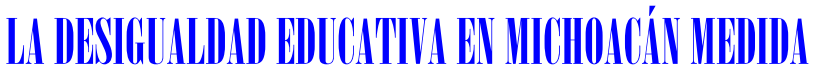

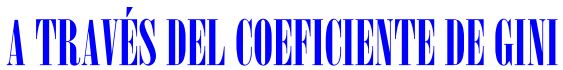

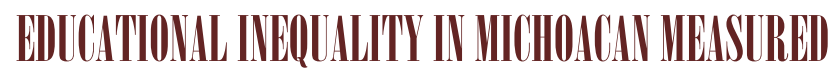

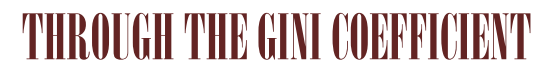

\author{
FAVILA TELLO Antonio \\ HERNÁNDEZ BARRIGA Plinio
}

Recepción: marzo 6 de 2019 | Aprobado para publicación: julio 12 de 2019

DOI: http://dx.doi.org/10.33010/ie_rie_rediech.v10i19.646

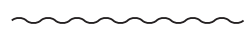

\section{Resumen}

La desigualdad educativa es uno de los principales problemas del ámbito educativo nacional dada su influencia en el ejercicio efectivo de los derechos de la población y sus implicaciones para la movilidad social. Pese a su importancia, el asunto ha sido poco diagnosticado en el ámbito estatal y menos aún para el ámbito municipal. El presente trabajo busca realizar un aporte en este sentido al cuantificar la desigualdad educativa en los municipios del estado de Michoacán a través del coeficiente de Gini para la educación. Los resultados sugieren la existencia de brechas de desigualdad significativas entre los diferentes

Antonio Favila Tello. Profesor-investigador del Instituto de Investigaciones Económicas y Empresariales de la Universidad Michoacana de San Nicolás de Hidalgo, México. Tiene estudios de contador público, maestro en ciencias, en comercio exterior y doctor en ciencias del desarrollo regional. Su desempeño profesional ha sido como consultor y en el sector público dentro del Ayuntamiento de Morelia, la Universidad Abierta y a Distancia de México y la Auditoría Superior de la Federación. Es miembro del Sistema Nacional de Investigadores Nivel Candidato y trabaja las líneas de investigación desigualdad educativa y economía de la innovación. Correo electrónico: antoniofavila@gmail. com. ID: http://orcid.org/0000-0001-8652-147X.

Plinio Hernández Barriga. Profesor-investigador del Instituto de Investigaciones Económicas y Empresariales de la Universidad Michoacana de San Nicolás de Hidalgo, México. Es licenciado en Economía por la misma universidad, maestro y doctor en Economía con Especialidad en Economía Financiera por la Universidad Nacional Autónoma de México. Es coordinador del Doctorado en Políticas Públicas y titular del proyecto de investigación "Factores económicos, institucionales y políticos que determinan la relación entre la productividad y la distribución del ingreso: un estudio comparado entre las economías industrializadas y emergentes". Ha publicado diversos artículos y capítulos de libros en temas relacionados con la economía financiera, distribución del ingreso y política económica. Correo electrónico: plinio@umich.mx. ID: http://orcid.org/0000-0002-7585-2721. 
municipios del estado, así como la concentración de los municipios más desiguales en la parte sur de la entidad. Dicha franja se extiende a través de la región costera del estado, abarcando desde los límites de Michoacán con el estado de Colima y ascendiendo hacia los límites con Guerrero y el Estado de México, encontrándose la mayor desigualdad en los municipios de Nocupétaro, Tumbiscatío y Carácuaro. El mapeo de estos datos puede coadyuvar a la toma de decisiones en la materia y a la localización de acciones compensatorias que colaboren para contrarrestar esta problemática.

\title{
Palabras clave: INEQUIDAD, MUNICIPAL, EDUCATIVA, ESCOLARIDAD, ÍNDICE.
}

\begin{abstract}
Educational inequality is one of the main problems of the national educational environment, given its influence on the effective exercise of population's rights and its implications for social mobility. Despite its importance, the matter has been underdiagnosed at the state level and even less for the municipal level. This paper seeks to contribute in this sense by quantifying educational inequality in the municipalities of Michoacán state, through the Gini coefficient for education. The results suggest the existence of significant inequality gaps between the different municipalities of the state, as well as the concentration of the most unequal municipalities in the southern part of the state; this zone extends through the coastal region of the state, ranging from the limits of Michoacán with Colima state and rising to the border with Guerrero and Estado de Mexico, finding the greatest inequality in the municipalities of Nocupétaro, Tumbiscatío and Carácuaro. The mapping of these data can contribute to the decision making in the matter and to locate compensatory actions that collaborate to counteract this problem.
\end{abstract}

Keywords: INEQUITY, MUNICIPAL, EDUCATIONAL, SCHOOL LEVEL, INDEX.

\section{IVTronencoriós}

\section{La desigualdad} ámbito educativo nacionaly un objeto de estudio recurrente desde disciplines como sociología, la demografía y la economía. De acuerdo con diversos estudios (Bracho, 1995; Martínez, 2002; Martínez, 1992; Navarro y Favila, 2013; Coneval, 2018), las brechas de desigualdad educativa que se experimentan entre las distintas regiones de México continúan y resisten el paso del tiempo en un ambiente en el que los recursos invertidos y las decisiones tomadas parecen aún insuficientes para generar cambios

140 disruptivos en dichas tendencias. 
La medición de la desigualdad educativa juega un papel importante en la toma de decisiones y en el diseño de políticas educativas, ya que permite dar seguimiento a las ganancias y pérdidas experimentadas por el sistema escolar, aproximarse a dimensionar la eficiencia de los recursos invertidos y tener una idea del aporte potencial de la educación a la productividad del país. Adicionalmente, estas mediciones permiten generar expectativas sobre las características de la fuerza de trabajo disponible y realizar previsiones acerca de algunos fenómenos asociados, tales como la desigualdad en la distribución del ingreso, el rezago educativo, la deserción escolar, el desempeño de los estudiantes en las pruebas de logro académico, la movilidad social y la prevalencia del analfabetismo funcional (Bracho, 1995; Martínez, 2002; Martínez, 1992; Tapia y Valenti, 2016; Torpey, 2018).

Dentro de las regiones que de acuerdo con la literatura del tema muestran una situación de rezago en comparación con el resto del país se encuentra el estado de Michoacán. Dicho estado constituye un caso particular de estudio donde coexisten diversos y contrastantes escenarios de desigualdad que se manifiestan en diferencias educativas sustanciales entre sus municipios. Pese a la importancia del tema, los trabajos recientes no parecen haber abordado de manera particular al estado de Michoacán ni a sus municipios en lo tocante a la desigualdad educativa.

De acuerdo con la información publicada por el INEGI (2015), Michoacán es el sexto estado del país con el mayor analfabetismo entre la población de 15 años y más (estando en esta condición 8 de cada 100 habitantes). Asimismo, Michoacán posee la cuarta escolaridad más baja del país (tomando en consideración a la población de 15 años y más) con solo 7.9 años. Adicionalmente, el estado obtuvo el lugar 31 de entre 32 entidades evaluadas por el índice de cumplimiento de la responsabilidad educativa (el cual evalúa el desempeño de las autoridades educativas locales) (Mexicanos Primero, 2018) e igualmente ocupó el lugar 31 de 32 en el índice de desempeño educativo incluyente (el cual evalúa el desempeño de los actores involucrados en la educación en seis dimensiones: aprendizaje, eficacia, permanencia, profesionalización, supervisión y participación) (Mexicanos Primero, 2014).

El presente trabajo, con intenciones mayormente descriptivas, pretende aportar elementos para la mejor comprensión de la desigualdad educativa para el caso del estado de Michoacán y coadyuvar en documentar el desempeño de sus municipios utilizando para ello una de las herramientas de medición típicas del tema: el coeficiente de Gini para la educación. A continuación, se realiza una revisión de la literatura del tema y del contexto estudiado; posteriormente se exponen los instrumentos de medición utilizados y los resultados encontrados, para cerrar con algunas conclusiones.

\section{RenISIÓN IE LITERITIRI}

Las nociones de igualdad y desigualdad hacen referencia, de manera general, a la distribución de un determinado bien entre los integrantes de una población; sin embargo, el concepto de igualdad educativa contiene una marcada subjetividad, ya que puede ser percibido de manera diferente de acuerdo con la perspectiva individual, familiar o social. Pese a la subjetividad que impregna al tema, la idea de la 
igualdad educativa hace referencia a la disponibilidad de oportunidades de acceso, permanencia, egreso y adquisición del nivel de aprendizaje esperado que disfrutan los miembros de una sociedad determinada (Martínez, 2002).

Para Juárez y Rodríguez (2016), la igualdad educativa implica que las desigualdades observadas se pueden atribuir solo a los méritos individuales; es decir, es un estado en el cual no existen o se han reducido al mínimo aquellos otros factores sociales, de cualquier índole, que pueden generar diferencias injustas en el nivel educativo de la población.

Por otro lado, para Bracho (1995), las diversas definiciones de la desigualdad educativa pueden clasificarse en dos grandes tipos. El primero se basa en los términos de la Declaración Universal de los Derechos Humanos de las Naciones Unidas (ONU, 1948), en la cual la igualdad educativa se refiere al acceso universal a la educación elemental y a la existencia de una oferta accesible de educación posbásica. El segundo tipo es de orden sociológico y considera que las expectativas de escolaridad responden a los estándares de cada grupo de referencia, por lo que no existe una norma absoluta de escolaridad ideal; los trabajos que adoptan este enfoque analizan la distribución de la educación en momentos o grupos relevantes para comprender su distribución precedente y establecer parámetros de expectativas plausibles. El presente trabajo se circunscribe a este segundo tipo.

De acuerdo con Schmelkes (2015), la configuración de la desigualdad educativa en México se explica a partir de dos grandes conjuntos de causas. El primero se refiere a las causas atribuibles al sistema escolar y el segundo a las condiciones del entorno.

Dentro del primer conjunto, parte de la problemática se relaciona con la manera en la que las autoridades educativas toman decisiones en la materia, situación en la que persisten malas prácticas administrativas, tales como la ocurrencia, la inercia, la opacidad, el nepotismo y la corrupción, las cuales merman el efecto de los recursos invertidos en el ámbito educativo. De manera adicional existe una cierta presión para que las autoridades educativas cumplan con determinados indicadores de desempeño; esta presión, en vez de servir como un mecanismo para la rendición de cuentas y la mejora de los procesos, suele tener un efecto adverso, ya que origina que se actúe con inmediatez y se maquillen cifras para dar cumplimiento a los requerimientos administrativos, situaciones que terminan generando información que no refleja adecuadamente la realidad y dificulta el diseño de políticas educativas sobre bases certeras (Fernández, 2015).

Otros problemas que generan un abordaje deficiente de la desigualdad educativa desde lo local se relacionan con la falta de claridad en la división de las responsabilidades entre los diferentes niveles de gobierno para la gestión y el uso de los recursos educativos. Esta situación ha provocado que los gobiernos locales hayan conservado funciones operativas generalmente condicionadas o sujetas a la supervisión del gobierno federal, viendo así limitada su capacidad de acción y generando una carencia de criterios, indicadores y normas que permitan una adecuada rendición de cuentas (México Evalúa, 2011). Estas carencias han generado que, pese a que en el sexenio 2012-2018 el gasto educativo fue equivalente al 14\% del presupuesto de egresos y a cerca del 3\% del PIB, el panorama educativo no mejoró de manera sustancial 142 (México Evalúa, 2018). 
A las situaciones anteriores hay que agregar la manera en la que se asigna el presupuesto educativo, la cual denota la ausencia de políticas de combate a la desigualdad y de corte compensatorio en las que se ofrezcan mayores recursos a aquellos que más los necesitan. En vez de seguir un criterio de igualdad, la asignación de dichos recursos obedece a la capacidad de negociación y de presión política de los actores involucrados, lo cual marca la pauta para que sean las zonas urbanas e industrializadas las que reciban mayores recursos en detrimento de las zonas predominantemente rurales, lo cual a su vez define las características y las modalidades de la oferta educativa local y profundiza las desventajas para los grupos poblacionales cuyas autoridades tienen una capacidad de interlocución política menor (Muñoz, 2009).

Observaciones similares pueden encontrarse en el trabajo de Schmelkes (2015), quien indica que el presupuesto en educación básica o media superior se distribuye de manera inercial, con lo cual se mantiene una presupuestación prácticamente uniforme de un ejercicio fiscal a otro con avances mínimos que apenas cubren los efectos de la inflación.

En este mismo sentido, México Evalúa (2011) señala que existe una marcada desconexión entre el gasto y los resultados obtenidos que sugiere una aplicación ineficiente de los recursos que tiene por consecuencia que ciertas áreas estratégicas para el avance del sistema educativo queden poco atendidas. Así, el hecho de que la mayor parte del gasto educativo se destine al pago de los aspectos salariales (alrededor del 97\% del total), deja solo algunos recursos marginales para realizar inversiones educativas que permitan el cierre de las brechas de desigualdad existentes. En este escenario, la estructura actual del gasto educativo no contempla la suficiencia presupuestaria necesaria para financiar la infraestructura, el equipamiento, la operación y el mantenimiento adecuado de los planteles. A lo anterior hace falta agregar las malas prácticas en la gestión del presupuesto educativo, tales como la financiación de actividades y pago de salarios a maestros en condiciones irregulares (comisionados, con plazas incompatibles o no registrados ante el Registro Federal de Contribuyentes) y el uso de los recursos con fines políticos (como la atención a protestas, manifestaciones y otras acciones disruptivas) (México Evalúa, 2018).

Esta situación se constata al revisar los datos del Censo de Escuelas, Maestros y Alumnos de Educación Básica y Especial (Cemabe) realizado durante el año 2013 y según el cual más de 40 mil escuelas se encontraban en construcciones precarias 0 adaptadas, cerca de 152 mil planteles ocupaban edificaciones de más de 30 años y requerían reparaciones mayores, más de 47 mil escuelas no cumplían con las especificaciones técnicas requeridas y alrededor de 10 mil carecían de paredes. Adicionalmente, de las escuelas públicas de educación básica del país, el 31\% carecía de agua de la red pública, el 48\% de drenaje, el 11\% de electricidad, el 13\% de baños, el 43\% de equipo de cómputo y el 69\% de internet (Márquez, 2015).

Estas dificultades presupuestarias pueden explicarse en primera instancia por las profundas diferencias existentes entre las aportaciones que realiza cada nivel de gobierno. El sistema educativo mexicano continúa siendo financiado principalmente por el gobierno federal; le siguen en importancia las aportaciones de los gobiernos estatales. Sin embargo, estas contribuciones son sumamente heterogéneas de un estado a otro y de un ejercicio fiscal al siguiente. Los gobiernos municipales, por su 
parte, realizan gastos en educación de manera muy escasa. Según estimaciones de México Evalúa (2011), del 100\% del gasto nacional en educación, un 64\% proviene del gobierno federal, un $21.6 \%$ del sector privado, un $14.3 \%$ de las entidades federativas y solo un $0.02 \%$ de los municipios.

De la misma forma, la manera de distribuir los recursos asume que los estudiantes de educación básica tienen las mismas necesidades en cualquier región del país, lo cual es impreciso. Esta manera de presupuestar omite el efecto de las profundas diferencias existentes de una entidad a otra, diferencias que a su vez representan costos diferenciados para educar; en consecuencia, no se provee de recursos suficientes que coadyuven a cerrar las brechas que separan a los estudiantes que se encuentran en situaciones de alta o muy alta marginación. Esta manera de presupuestar termina provocando que los alumnos que se encuentran en una mejor situación tengan acceso a mayores recursos de manera constante, lo que perpetúa y explica la brecha de desigualdad entre ellos (México Evalúa, 2011).

Lo anterior representa una pauperización de la educación, ya que los incrementos en la matrícula no se ven compensados con aumentos proporcionales en los recursos invertidos en la misma. Esto no genera un esquema en el que los recursos se utilicen de manera más eficiente, sino que, por el contrario, se sacrifica la calidad educativa para conseguir una sobreexplotación de los recursos asignados (Muñoz, 2009).

El segundo conjunto de causas se refiere a aquellas atribuibles al entorno. Estas explican que las diferencias regionales en materia de desigualdad educativa obedecen a una diversidad de razones históricas y culturales que definieron los diversos escenarios de desarrollo económico existentes en México, tales como la concentración de las actividades económicas en el centro del país y sus estados circundantes y las grandes migraciones producto de dicha concentración. Los movimientos migratorios derivados de estos procesos generaron que algunas entidades mejoraran en sus indicadores de distribución de la escolaridad por causa de la relocalización de los recursos humanos especializados en las regiones industrializadas, las zonas productoras de hidrocarburos y alrededor de las grandes universidades y centros de investigación (Martínez, 1992). Con el paso de los años, la zona metropolitana de la Ciudad de México se consolidó como el mayor centro atractor de migrantes, con la adición de algunas ciudades medias en la frontera con Estados Unidos y en las regiones turísticas del Caribe mexicano. De esta forma, la migración de la ciudad al campo se sustituyó por un patrón migratorio de ciudad a ciudad, lo cual tendió a profundizar las brechas educativas entre lo rural y lo urbano (Pérez y Santos, 2013).

Otro aspecto que explica las diferencias regionales en materia de desigualdad educativa es el tocante a la cultura. En México, a diferencia de lo ocurrido en países como Chile, Uruguay o Argentina, la legislación sobre la obligatoriedad de la educación básica se dio cuando en el país una gran cantidad de la población adulta aún era analfabeta; 10 anterior provocó que muchas familias no valoraran adecuadamente los beneficios sociales, psicológicos y formativos de la educación, lo cual, aunado a la escasez de recursos, generó que la educación de los hijos no fuera considerada como una prioridad en muchos hogares (Martínez, 1992). A esto es necesario sumar la incidencia que aspectos como las diferencias generacionales y el género pueden 
ejercer sobre la desigualdad educativa, particularmente en las zonas rurales más vulnerables y empobrecidas (Bracho, 1995).

La influencia del entorno alcanza también a la manera en que las personas se apropian de los contenidos que se les imparten. Los aprendizajes se ven afectados por la desconexión existente entre las necesidades de los sectores más desfavorecidos y los contenidos curriculares, los cuales son diseñados centralmente, omitiendo las necesidades, intereses y vocaciones locales y perpetuando la visión de dominio y subordinación que existen tanto entre países centrales y periféricos como entre regiones dentro de un mismo país (Muñoz, 2009).

A estas carencias del entorno se suman las deficiencias en el diseño de los agentes encargados de impartir educación entre la población más vulnerable y a las deficiencias en la formación de los recursos humanos destinados a dichas tareas. Es frecuente que por estas causas los atrasos pedagógicos que experimentan los estudiantes menos favorecidos sean tratados con indiferencia y no se establezcan estrategias dentro del aula que permitan compensar estas situaciones. El abordaje deficiente de estas diferencias incluso coadyuva a profundizarlas, generando sentimientos de minusvaloración entre los estudiantes e incentivando la deserción escolar temprana. La propia falta de recursos de estas escuelas implica que no cuenten con alternativas que reviertan estos patrones en los que los maestros tienden a responsabilizar del bajo rendimiento escolar a las familias, las familias a los maestros y los estudiantes a ellos mismos (Muñoz, 2009).

Al respecto, Backhoff et al. (2007) señalan que los factores que más impactan en este sentido son las características de los estudiantes, las singularidades de sus familias, la modalidad educativa (si la escuela es privada, urbana, rural, indígena o comunitaria) y los factores de composición de las escuelas, seguidos por las propiedades estructurales de los centros escolares. Señalan también que la distancia de desigualdad en el aprendizaje escolar entre las escuelas más avanzadas y las más rezagadas es de entre 3 y 4 grados escolares y que la modalidad educativa explica entre el 48 y el $61 \%$ de dicha variación.

De manera similar, Schmelkes (2015) identifica las siguientes condiciones de entorno como determinantes significativos de la desigualdad educativa para el caso de México: a) habitar en una zona rural o urbana; b) la pertenencia a algún grupo indígena; c) habitar en zonas identificadas como de alta o muy alta marginación; d) la escolaridad de los padres; e) el ingreso familiar; f) la incorporación temprana al mercado laboral; $\mathrm{y}, \mathrm{g}$ ) la importancia otorgada a la escolaridad en el seno familiar y las expectativas acerca de cómo la educación puede generar una diferencia en su calidad de vida.

Las causas de entorno influyen también en el mercado laboral de los docentes y en la forma en la que se organizan. Dicho mercado no es homogéneo a lo largo del territorio nacional y se encuentra integrado de al menos tres grandes estratos: el urbano de las clases medias y altas, el urbano marginado y el rural. Esta estratificación genera amplios incentivos no salariales para los maestros ubicados en zonas urbanas, los cuales tienen un acceso más fácil a los servicios. Al no existir un sistema que compense estos incentivos para el caso de los maestros rurales, estos tienden a solicitar ser trasladados a las ciudades, situación que tiene por consecuencia que las 
escuelas rurales sean generalmente atendidas por docentes de menor experiencia 0 de menor capacidad de gestión (Muñoz, 2009).

Lo anterior es evidente en el caso de la educación comunitaria, la cual es atendida generalmente por jóvenes estudiantes de bachillerato, los cuales se encuentran expuestos no solo a las dificultades que les impone el encontrarse en comunidades de difícil acceso, sino también a los peligros de la delincuencia que opera en dichas zonas, situación que genera una amplia deserción de los docentes de esta modalidad (Cano, 2016).

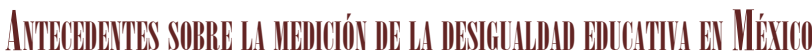

Uno de los aspectos más polémicos alrededor del estudio de la desigualdad educativa es el relativo a su medición, lo cual plantea tanto dificultades instrumentales como teóricas. En opinión de Bracho (1995), la medición cuantitativa de la desigualdad educativa enfrenta tres grandes problemas: 1) generalmente se cuenta solo con la información relacionada con la escolaridad, es decir con la obtención de credenciales educativas por parte de la población, las cuales no necesariamente reflejan los conocimientos adquiridos durante la trayectoria escolar; 2) la escolaridad es acumulable, y una vez que se adquiere ya no puede perderse, lo que implica que la acumulación de la escolaridad en algunos segmentos de la población no representa pérdidas para los otros segmentos, lo cual dificulta el uso de algunos instrumentos estadísticos y su interpretación; y, 3) existe una carencia de criterios normativos para evaluar a la desigualdad educativa, es decir, la interpretación de su medición depende de su evolución en el tiempo o de la comparación entre entidades, así como de lo que la sociedad considere como justicia distributiva en un determinado momento histórico.

Nuevas ideas han emergido para contrarrestar estas dificultades y para encontrar fuentes alternativas de datos para realizar este tipo de análisis, por ejemplo midiendo a la desigualdad educativa en términos absolutos y relativos, midiendo cohortes de población y su evolución a través del tiempo e incorporando indicadores generados en las pruebas de logro académico y en sus cuestionarios de contexto (Bruckauf y Chzhen, 2016); sin embargo, el interés por la medición de la desigualdad educativa a partir de indicadores de escolaridad ha permanecido por su versatilidad y facilidad de interpretación.

Martínez (1992) señala que la falta de información censal periódica constante dificulta la comparabilidad de las mediciones sobre desigualdad educativa, ya que los datos disponibles para un determinado grupo etario pueden estar disponibles para un determinado censo y no estarlo para los siguientes o los anteriores. Algo similar sucede con la manera en la que se dividen los grados educativos en los censos de población, en los cuales ocasionalente existe información con una mayor desagregación, la cual puede no ser publicada de la misma forma en emisiones posteriores. A esto hay que adicionar que el valor de un coeficiente de desigualdad solo puede interpretarse en función de la comparación; es decir, de los resultados obtenidos en otros casos, por lo que su análisis requiere de un cierto conocimiento 146 empírico de la situación predominante. 
La desigualdad educativa en México ha sido medida tradicionalmente a través de la escolaridad promedio y la tasa de analfabetismo, las cuales se abastecen generalmente con los resultados de los censos y conteos de población y vivienda. Además de estas medidas, existen registros de la medición del coeficiente de Gini para la educación que documentan la situación nacional a partir de la segunda mitad del siglo XX.

A partir de 1966, el debate sobre la desigualdad educativa y la manera en que debería medirse recibe un impulso importante a partir de la publicación del Informe Coleman, el cual fue un trabajo pionero que ponía en tela de juicio las razones por las cuales aquellos alumnos que se encontraban en condiciones sociales desfavorables (principalmente pertenecientes a minorías y grupos raciales segregados) tendían a obtener los peores resultados escolares en los Estados Unidos. Previo al Informe Coleman, la relación entre la educación y la posición social de las personas no era generalmente aceptada entre los académicos y las diferencias en el desempeño de los alumnos solían atribuirse a causas genéticas y a malos hábitos individuales. El Informe Coleman planteó la necesidad de definir a la desigualdad educativa y puso un gran énfasis en la necesidad de su diagnóstico y de la toma de decisiones deliberadas para su diminución (Marqués, 2016).

En los años posteriores, diversos trabajos abordaron el tema de manera empirista y cuantitativa. Para el caso de México, Martínez (1992) realiza uno de los trabajos representativos del tema, dando cuenta de la disminución de la tasa de analfabetistmo en adultos durante el siglo xx, la cual pasó del $81.5 \%$ en 1910 al 9.9\% en 1985. Otro aporte del mismo trabajo es el cálculo del coeficiente de Gini para la educación en México, el cual pasó de 0.56 en 1950 a 0.39 en 1990.

Martínez (1992) realiza también la medición del coeficiente de Gini para la educación por entidad federativa para los años 1970, 1980 y 1990. Los resultados obtenidos por el autor ponen en evidencia las profundas brechas de desigualdad existentes entre los estados del país y la manera en la que dichas brechas resisten a pesar del paso del tiempo.

En 1970, el coeficiente de Gini entre los estados de la República Mexicana fue de entre 0.69 y 0.38 ; en 1980 , fue de entre 0.58 y 0.28 y en 1990 de entre 0.54 y 0.29. Las entidades más rezagadas en los tres años calculados (1970, 1980 y 1990) son Chiapas, Oaxaca y Guerrero. Michoacán es el cuarto peor evaluado en 1970 y el sexto peor evaluado en 1980 y 1990. Las entidades mejor evaluadas en los tres años calculados son el Distrito Federal, Nuevo León y Baja California. La brecha de desigualdad es tan persistente y profunda que se estima que le tomaría cuarenta años a la entidad más atrasada alcanzar a la más igualitaria. Estos resultados manifiestan que, pese a los avances obtenidos por las entidades, las brechas de desigualdad educativa entre ellas son amplias, resisten el paso del tiempo y avanzan de manera inercial a consecuencia del proceso de modernización (Martínez, 1992).

Con posterioridad a este estudio, Martínez (2002) realiza una nueva medición del coeficiente de Gini para la educación con datos estatales para el año 2000. En esta ocasión, el índice de Gini para la entidad más igualitaria es de 0.25 , mientras que el más alto obtenido es de 0.48 . Nuevamente las entidades con coeficientes superiores 
son Chiapas, Oaxaca y Guerreo; les siguen en importancia Veracruz y Michoacán. Las entidades más igualitarias fueron el Distrito Federal, Nuevo León y Coahuila.

Salgado y Rodríguez (2012) realizan cálculos parecidos a los anteriores con datos para el año 2005. Sus resultados sugieren que las entidades más desiguales en materia de educación fueron Chiapas, Puebla, Oaxaca, Guerrero y Michoacán, mientras que las más igualitarias resultaron ser Quintana Roo y el Estado de México.

En un estudio similar, Navarro y Favila (2013) miden los coeficientes de Gini para la educación con datos estatales para 2010. En concordancia con lo publicado por Martínez (2002), nuevamente las entidades más desiguales son Chiapas, Guerrero y Oaxaca, seguidas por Michoacán y Veracruz. Algo parecido puede observarse en el trabajo de Favila y Navarro (2017), donde los autores calculan estos coeficientes con datos para el año $2015 \mathrm{y}$ encuentran que nuevamente las entidades con los coeficientes de Gini más altos fueron Chiapas (con 0.32), Guerrero (con 0.319, Oaxaca (con 0.29), Veracruz (con 0.26) y Michoacán (con 0.26). Las condiciones más favorables se encontraron nuevamente en el Distrito Federal (0.17), Nuevo León (0.18) y Sonora (0.19).

Como puede apreciarse, salvo por pocos cambios las entidades más igualitarias y las menos igualitarias continúan siendo practicamente las mismas, al menos desde 1970 y hasta 2015. Entre los resultados de estos estudios destaca la frecuencia con la que Michoacán se ha ubicado en las partes más bajas de estas clasificaciones a través de los años.

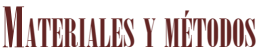

El coeficiente de Gini es un indicador creado originalmente para medir las desigualdades económicas (particularmente la de la distribución del ingreso), aunque puede utilizarse para medir cualquier forma de distribución desigual. Matemáticamente, el coeficiente de Gini es la razón de la mitad del promedio sobre todos los pares de las absolutas deviaciones, entre todos los posibles pares de personas (Salgado y Rodríguez, 2012).

Para el cálculo del coeficiente de Gini para la educación se retoma la fórmula utilizada por Thomas et al. (2001), la cual es la siguiente:

$$
E L=\left(\frac{1}{\mu}\right) \sum_{i=2}^{n} \sum_{j=1}^{i-1} P_{i}\left|Y_{i}-Y_{j}\right| P_{j}
$$

Donde:

$E L$ : Representa el coeficiente de Gini para la educación; es decir, el grado de desigualdad existente en materia educativa entre los miembros de la población, considerando como criterio la distrbución de la escolaridad.

$\mu$ : Es el promedio de años de escolaridad para la población estudiada.

$n$ : Es el número de divisiones en las que se segmentan los niveles de escolaridad de una sociedad. En este caso, la Encuesta Intercensal del INEGI (2015) divide 
la escolaridad de la población en siete categorías: sin escolaridad, preescolar, primaria completa, secundaria incompleta, secundaria completa, media superior v siperior.

$P_{i}$ y $P_{j}$ : Representan las proporciones de la población que posee un nivel de educación determinado; aquí, alguna de las siete categorías mencionadas en la definición de $n$.

$Y_{i}$ y $Y_{j}$ : Representan el número de años de escolaridad requeridos para alcanzar un nivel de educación determinado. Para este caso se asignaron los siguientes: sin escolaridad, cero años; preescolar, 3 años; primaria completa, 9 años; secundaria incompleta, 10.5 años; secundaria completa, 12 años; educación media superior, 15 años; y educación superior, 20 años.

El coeficiente de Gini puede alcanzar valores entre 0 y 1 , donde 0 representa la perfecta igualdad y 1 representa la perfecta desigualdad. Los valores más cercanos a cero indican una distribución de la escolaridad más igualitaria (Thomas et al., 2001).

Los cálculos se realizaron con base en los resultados de la Encuesta Intercensal 2015 publicada por el INEGI. Utilizando la fórmula descrita se calcularon los coeficientes de Gini para la educación de los 113 municipios del estado de Michoacán para la población de 15 años y más. Los resultados obtenidos se muestrana continuación.

\section{Resilitillos}

La tabla 1 muestra los resultados obtenidos para el coeficiente de Gini para la educación en los municipios del estado de Michoacán. El valor promedio de los 113 municipios que conforman el estado fue de 0.243 , con una desviación estandar de 0.0385 , lo que sugiere que, para el caso de la población de 15 años y más, la desigualdad educativa medida por la distribución de la escolaridad muestra una baja dispersión. El valor mínimo obtenido fue de 0.189 y el máximo de 0.375 .

Cabe destacar que 39 (34\%) de los 113 municipios se encontraron por debajo de la media para este indicador. Los municipios más igualitarios del estado fueron Nuevo Parangaricutiro, Charo, Ocampo, Ziracuaretiro y Purépero. Los más desiguales en materia educativa fueron Nocupetaro, Tumbiscatío, Carácuaro, San Lucas y Tiquicheo. Morelia, la capital del estado, ocupó el puesto número 9 de la clasificación.

Estos datos también se utilizaron para la elaboración del mapa 1, cuya finalidad fue identificar si los municipios con la mayor desigualdad educativa guardaban un patrón geográfico particular. Para ello se dividieron los resultados obtenidos en tres rangos. Los municipios en blanco obtuvieron coeficientes entre 0.18 y 0.251 , los que se encuentran en gris claro entre 0.254 y 0.31 y los que se encuentran en gris oscuro mostraron coeficientes superiores a 0.32 .

La distribución municipal de los coeficientes de Gini más altos revela que las zonas más desiguales en materia educativa tienden a concentrarse alrededor de los límites entre Guerrero y Michoacán, incluyendo también a los municipios costeros del estado, formando una franja que se extiende desde los límites con Colima hasta los límites con el Estado de México. Pese a encontrarse influido por dicha zona, el 
Tabla 1. Coeficientes de Gini para la población de 15 años y más en los municipios del estado de Michoacán

\begin{tabular}{|c|c|c|c|c|c|c|c|c|}
\hline No. & Municipio & $\begin{array}{l}\text { Gini } \\
2015\end{array}$ & No. & Municipio & $\begin{array}{l}\text { Gini } \\
2015\end{array}$ & No. & Municipio & $\begin{array}{l}\text { Gini } \\
2015\end{array}$ \\
\hline 1 & $\begin{array}{l}\text { Nuevo } \\
\text { Parangaricutiro }\end{array}$ & 0.189 & 39 & Coeneo & 0.224 & 77 & Nuevo Urecho & 0.248 \\
\hline 2 & Charo & 0.190 & 40 & Peribán & 0.224 & 78 & Paracho & 0.248 \\
\hline 3 & Ocampo & 0.192 & 41 & Panindícuaro & 0.225 & 79 & Apatzingán & 0.248 \\
\hline 4 & Ziracuaretiro & 0.192 & 42 & Tzintzuntzan & 0.225 & 80 & Senguio & 0.251 \\
\hline 5 & Purépero & 0.197 & 43 & La Piedad & 0.225 & 81 & Parácuaro & 0.254 \\
\hline 6 & Angangueo & 0.201 & 44 & Penjamillo & 0.227 & 82 & Susupuato & 0.254 \\
\hline 7 & Huiramba & 0.201 & 45 & Tanhuato & 0.227 & 83 & Chinicuila & 0.258 \\
\hline 8 & Indaparapeo & 0.201 & 46 & Taretan & 0.227 & 84 & Juárez & 0.258 \\
\hline 9 & Morelia & 0.201 & 47 & Aporo & 0.227 & 85 & Buenavista & 0.261 \\
\hline 10 & Jungapeo & 0.202 & 48 & Tingambato & 0.228 & 86 & Tepalcatepec & 0.265 \\
\hline 11 & Tarímbaro & 0.203 & 49 & Morelos & 0.228 & 87 & Múgica & 0.267 \\
\hline 12 & Lagunillas & 0.203 & 50 & $\begin{array}{l}\text { Cojumatlán de } \\
\text { Régules }\end{array}$ & 0.229 & 88 & Epitacio Huerta & 0.269 \\
\hline 13 & Churintzio & 0.203 & 51 & Tingüindín & 0.230 & 89 & Aquila & 0.270 \\
\hline 14 & Briseñas & 0.203 & 52 & $\begin{array}{l}\text { Salvador } \\
\text { Escalante }\end{array}$ & 0.230 & 90 & Huandacareo & 0.271 \\
\hline 15 & Tuxpan & 0.204 & 53 & Erongarícuaro & 0.231 & 91 & Nahuatzen & 0.271 \\
\hline 16 & $\begin{array}{l}\text { Marcos } \\
\text { Castellanos }\end{array}$ & 0.206 & 54 & $\begin{array}{l}\text { José Sixto } \\
\text { Verduzco }\end{array}$ & 0.232 & 92 & Santa Ana Maya & 0.272 \\
\hline 17 & Tlalpujahua & 0.207 & 55 & Zamora & 0.233 & 93 & Tocumbo & 0.272 \\
\hline 18 & Queréndaro & 0.208 & 56 & Ecuandureo & 0.233 & 94 & Tangamandapio & 0.273 \\
\hline 19 & Zacapu & 0.211 & 57 & Tancítaro & 0.234 & 95 & Coahuayana & 0.274 \\
\hline 20 & Lázaro Cárdenas & 0.213 & 58 & Chucándiro & 0.234 & 96 & Gabriel Zamora & 0.276 \\
\hline 21 & Tlazazalca & 0.214 & 59 & Tangancícuaro & 0.235 & 97 & Madero & 0.276 \\
\hline 22 & Yurécuaro & 0.215 & 60 & Zitácuaro & 0.235 & 98 & Charapan & 0.277 \\
\hline 23 & Sahuayo & 0.215 & 61 & Contepec & 0.235 & 99 & Tzitzio & 0.282 \\
\hline 24 & Álvaro Obregón & 0.215 & 62 & Chilchota & 0.236 & 100 & Churumuco & 0.287 \\
\hline 25 & Huaniqueo & 0.215 & 63 & Quiroga & 0.236 & 101 & Coalcomán & 0.288 \\
\hline 26 & Ixtlán & 0.216 & 64 & Pajacuarán & 0.238 & 102 & La Huacana & 0.289 \\
\hline 27 & Hidalgo & 0.216 & 65 & Puruándiro & 0.238 & 103 & Cotija & 0.291 \\
\hline 28 & Jiménez & 0.218 & 66 & Jiquilpan & 0.239 & 104 & Tuzantla & 0.295 \\
\hline 29 & Angamacutiro & 0.218 & 67 & Irimbo & 0.240 & 105 & Arteaga & 0.319 \\
\hline 30 & Uruapan & 0.218 & 68 & Ario & 0.240 & 106 & Aguililla & 0.321 \\
\hline 31 & Chavinda & 0.218 & 69 & Maravatío & 0.241 & 107 & Turicato & 0.330 \\
\hline 32 & Numarán & 0.219 & 70 & Cherán & 0.242 & 108 & Huetamo & 0.330 \\
\hline 33 & Acuitzio & 0.220 & 71 & Cuitzeo & 0.242 & 109 & Tiquicheo & 0.340 \\
\hline 34 & Vista Hermosa & 0.220 & 72 & Jacona & 0.243 & 110 & San Lucas & 0.345 \\
\hline 35 & Pátzcuaro & 0.220 & 73 & Zináparo & 0.243 & 111 & Carácuaro & 0.348 \\
\hline 36 & Copándaro & 0.221 & 74 & Los Reyes & 0.243 & 112 & Tumbiscatío & 0.366 \\
\hline 37 & $\begin{array}{l}\text { Venustiano } \\
\text { Carranza }\end{array}$ & 0.223 & 75 & Villamar & 0.244 & 113 & Nocupétaro & 0.375 \\
\hline 38 & Zinapécuaro & 0.223 & 76 & Tacámbaro & 0.247 & & & \\
\hline
\end{tabular}

Fuente: Elaboración propia, con datos de INEGI (2015). 
municipio de Lázaro Cárdenas parece haber escapado de la tendencia de los municipios que lo circundan. Las condiciones más favorables pueden ubicarse en el centro y norte del estado, así como en los límites de Michoacán con Jalisco y la mayor parte de los límites con Guanajuato.

Mapa 1. Coeficientes de Gini para la educación en los municipios del estado de Michoacán

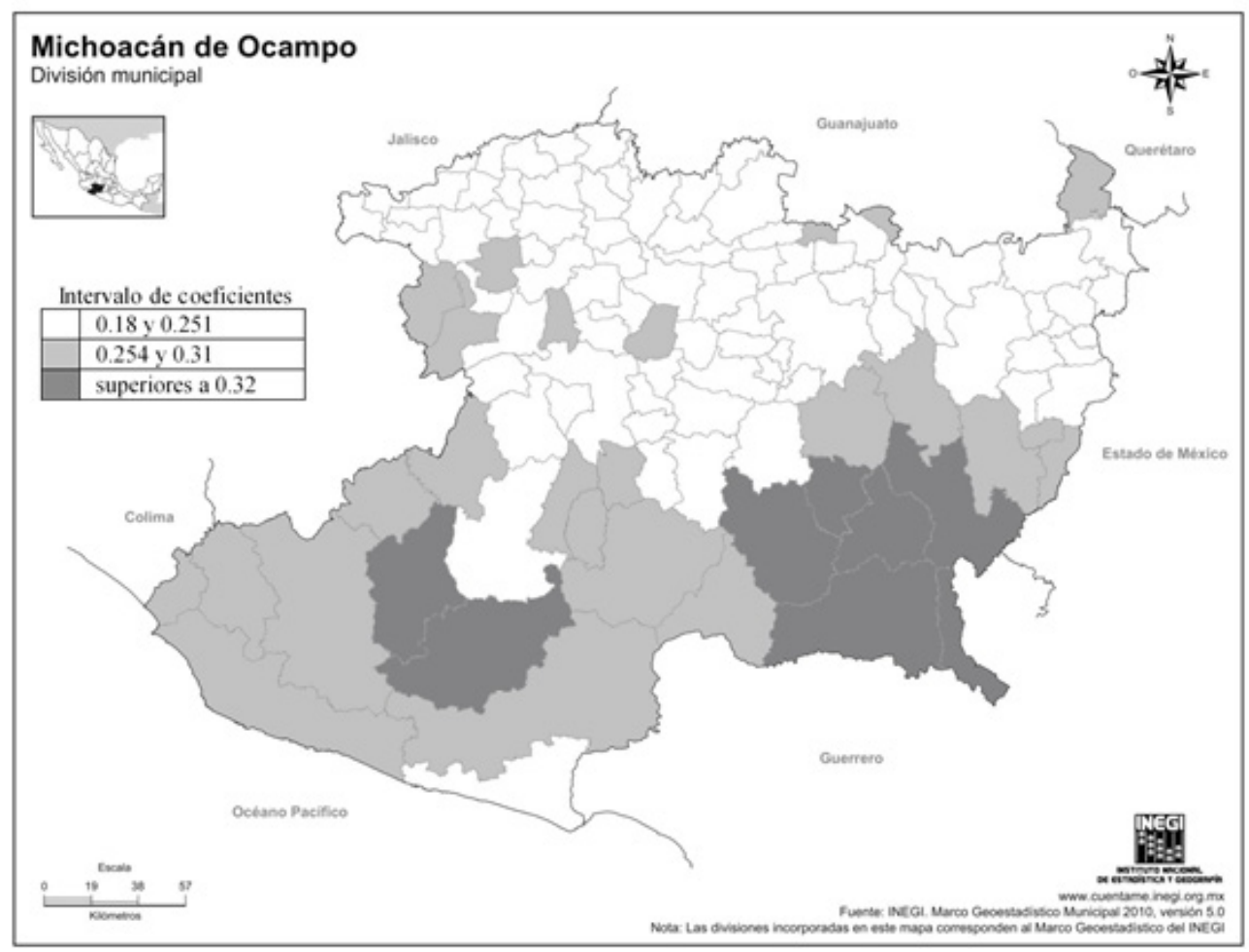

Fuente: Elaboración propia.

\section{Conchisiones}

La desigualdad educativa y su cuantificación son temas de relevancia para el diseño de políticas educativas, para la evaluación del desempeño del sistema educativo y para documentar la evolución histórica del fenómeno. Por ello consideramos relevante la utilización del coeficiente de Gini para seguir generando indicadores sobre el tema, tal como se hizo durante la segunda mitad del siglo XX.

El presente trabajo realiza un aporte sobre los estudios que diagnostican la desigualdad educativa a una escala municipal, los cuales aún son escasos, comparados con el nivel estatal y nacional. Tomando el caso del estado de Michoacán, hemos identificado brechas significativas en el desempeño de sus 113 municipios mediante el cálculo del coeficiente de Gini, las cuales mostramos en un patrón geográfico que posteriormente puede ayudar a localizar estrategias que coadyuven a disminuirlas.

Consideramos que las futuras líneas de investigación deberían abundar en la medición de la forma en la que las características de cada municipio explican su desempeño en materia de desigualdad educativa, así como sobre el impacto de la 
desigualdad en otros fenómenos sociales. Adicionalmente, es importante el diseño de alternativas que permitan solventar las carencias de información municipal periódica que obstaculizan la aplicación de otros instrumentos estadísticos que permitan la elaboración de un mayor número de trabajos de corte correlacional y explicativo.

Los resultados del presente trabajo, aunque descriptivos, permiten identificar distinciones claras entre el Michoacán urbano del norte y centro y el Michoacán rural, del sur y la costa. Las zonas del norte y centro del estado, donde se encuentran los centros urbanos importantes, la infraestructura de conectividad con las ciudades importantes del país, las zonas que lograron vincularse a la producción de bienes destinados a los mercados internacionales y las regiones que reciben influencia de los estados industrializados del centro y del Bajío muestran ventaja sobre el Michoacán del sur y la costa, territorios caracterizados por la producción de bienes agrícolas para el mercado nacional y las carencias de infraestructura educativa y de comunicaciones.

Una mirada breve a los indicadores constata lo anterior. Las zonas rezagadas en materia educativa coinciden con aquellas de mayor marginación, mayor incidencia de pobreza y mayor desigualdad en la distribución del ingreso (Conapo, 2016; Coneval, 2011, 2016). Son también zonas en las que la delincuencia organizada ha encontrado lugares fértiles, aislados y abundantes en trabajadores jóvenes, empobrecidos y carentes de alternativas para desarrollar sus actividades.

En este escenario, el desconocimiento de la evolución del sistema educativo en el estado juega un papel activo en la perpetuación de las desigualdades sociales y en el acentuamiento de los problemas locales. Es necesario que los estudios de este tipo sean tomados en consideración al momento de decidir el destino de los recursos, tomando en cuenta que utilizarlos bajo un estricto criterio de costo-beneficio condena a estas regiones a una desventaja permanente.

\section{RefrererenIs}

Backhoff, E., Bouzas, A., Contreras, C., Hernández, E. y García, M. (2007). Factores escolares y aprendizaje en México. México: INEE.

Bracho, T. (1995). Distribución y desigualdad educativa en México. Estudios Sociológicos, 13(37), 25-53.

Bruckauf, Z. y Chzhen, Y. (2016). Education for all? Measuring inequality of educational outcomes among 15-years-olds across 39 industrialized nations. Florencia: UNICEF.

Cano, A. (2016). Jóvenes instructores de Conafe se enfrentan a diversos riesgos y son víctimas en peligro. Diario Provincia. Recuperado de http://www.provincia.com.mx/ web/J\%C3\%B3venes_instructores_de_Conafe_se_enfrentan_a_diversos_riesgos_y_ son_v\%C3\%ADctimas_de_peligro-35993

Consejo Nacional de Evaluación de la Política de Desarrollo Social, Coneval. (2011). Indicadores de desigualdad. Base de datos. Recuperado de https://www.coneval.org.mx/coordinacion/ entidades/Michoacan/Paginas/desigualdad.aspx

Consejo Nacional de Evaluación de la Política de Desarrollo Social, Coneval. (2016). Resultados de pobreza en México 2016 a nivel nacional y por entidades federativas. Base de datos. Recuperado https://www.coneval.org.mx/Medicion/MP/Paginas/Pobreza_2016.aspx

Consejo Nacional de Evaluación de la Política de Desarrollo Social (CONEVĀL) (2018) Estudio Diagnóstico del Derecho a la Educación 2018. Ciudad de México: CONEVAL. 
Consejo Nacional de Población, Conapo. (2016). Datos abiertos del índice de marginación. Base de datos. Recuperado de http://www.conapo.gob.mx/es/CONAPO/Datos_Abiertos del Indice de Marginacion

Favila, A. y Navarro, J. (2017). Desigualdad educativa y su relación con la distribución del ingreso en los estados mexicanos. CPU-e Revista de Investigación Educativa, 24(1), 75-98.

Fernández, M. (2015). Desigualdades educativas: el panorama educativo de México. Animal Político. Recuperado de http://www.animalpolitico.com/blogueros-el-blog-de-mexicoevalua/2015/11/02/desigualdades-educativas-el-panorama-educativo-de-mexico

INEGI. (2015). Encuesta intercensal 2015 tabulados predefinidos. Base de datos. Recuperado de http://www.beta.inegi.org.mx/proyectos/enchogares/especiales/intercensal/

Juárez, D. y Rodríguez, C. (2016). Factores que afectan la equidad educativa en escuelas rurales de México. Pensamiento Educativo, 53(2), 1-15.

Marqués, I. (2016). Apuntes sobre el Informe Coleman. Sobre la difícil convivencia de los principios igualitario en un mundo desigual. International Journal of Sociology of Education, $5(2), 107-126$.

Márquez, A. (2015). El costo del derecho a la educación. Perfiles Educativos, 37(150), 3-17.

Martínez, F. (1992). La desigualdad educativa en México. Revista Latinoamericana de Estudios Educativos, 22(2), 59-120.

Martínez, F. (2002). Nueva visita al país de la desigualdad. La distribución de la escolaridad en México, 1970-2000. Revista Mexicana de Investigación Educativa, 7(16), 415-443.

Mexicanos Primero. (2014). Índice de desempeño educativo incluyente. México: Mexicanos Primero.

Mexicanos Primero. (2018). Índice de cumplimiento de la responsabilidad educativa. México: Mexicanos Primero.

México Evalúa. (2011). 10 puntos para entender el gasto educativo en México: consideraciones sobre su eficiencia. México: México Evalúa.

México Evalúa. (2018). Léase si quiere gobernar (en serio). México: México Evalúa, CIDAC.

Muñoz, C. (2009). Construcción del conocimiento sobre la etiología del rezago educativo y sus implicaciones para la orientación de las políticas públicas: la experiencia de México. Revista Iberoamericana sobre Calidad, Eficacia y Cambio en Educación, 7(4), 28-45.

Navarro, J. y Favila, A. (2013). La desigualdad de la educación en México, 1990-2010: el caso de las entidades federativas. Revista Electrónica de Investigación Educativa, 15(2), 21-33.

ONU. (1948). Declaración Universal de los Derechos Humanos. Nueva York: Organización de las Naciones Unidas.

Pérez, E. y Santos, C. (2013). Tendencias recientes de la migración interna en México. Papeles de Población, 76(2), 53-88.

Salgado, J. y Rodríguez, K. (2012). La desigualdad educativa en México por entidad federativa 1995-2005. Revista Educación, 36(1), 45-62.

Schmelkes, S. (2015). La desigualdad educativa en México. Recuperado de http://innovec.org.mx/ home/images/2-sschmelkes.pdf

Tapia, L. y Valenti, G. (2016). Desigualdad educativa y desigualdad social en México. Nuevas evidencias desde las primarias generales en los estados. Perfiles Educativos, 38(151), 32-54.

Thomas, V., Wang, Y. y Fan, X. (2001). Measuring education inequality: Gini coefficients of education. Washington: World Bank.

Torpey, N. (2018). Measuring education inequality in developing countries. Berlín: Springer. 
